\title{
DEVELOPING A LIBRARY TOOLBAR
}

Recently, there has been a movement in the library community to embrace Web 2.0 technologies such as instant messaging, social networking, and blogging. Among these technologies is the library toolbar. Modeled after the Google and Yahoo toolbars, the library toolbar is embedded in the web browser and allows users to search the library online public access catalog (OPAC) and online databases from any web site. While this is the main feature, the toolbar can also contain quick links to the library's homepage, reserves, research guides, operating hours, and reference services.

Oklahoma City University's Dulaney-Browne Library (DBL) serves an estimated population of 5500 with a staff of seventeen. In the last few years DBL has tried several ways to market services to patrons. The web site was redesigned and a logo for the library was created, a monthly newsletter highlighting events in the library was started, and a marketing plan implemented. When Conduit ${ }^{\mathrm{TM}}$ (www.conduit.com) was mentioned in the recent Computers in Libraries article, "Online Treasures" (Balas, 2008), it was seen as another opportunity to market library services. Conduit ${ }^{\mathrm{TM}}$ is one of several options available to create a custom toolbar. Instantbar (www.instantbar.com), Bizbar (www.bizbar.com), Toolbar Browser (www.toolbarbrowser.com), and Conduit ${ }^{\mathrm{TM}}$ were all evaluated as possible services for creating a library toolbar. Conduit ${ }^{\mathrm{TM}}$ was chosen because it was the only service that was compatible with both Internet Explorer and Mozilla Firefox in addition to being free. An added bonus is that Conduit ${ }^{\mathrm{TM}}$ is powered by Google. This article will chronicle the experiences of the DulaneyBrowne Library in the development of a customized Conduit ${ }^{\mathrm{TM}}$ toolbar.

\section{Developing the Toolbar}

The first step in developing a Conduit ${ }^{\mathrm{TM}}$ toolbar is signing up for an account. A few pieces of information are required to complete this process such as an organizational web address. Adding a menu of links or RSS feeds are other options available during the registration process. Being unfamiliar with the process, most pieces of information were left blank and filled in during the customization process. At the end of the registration process the library toolbar is created and customization can be started.

The search box was the Dulaney-Browne Library's (DBL) main focus as it is the defining feature of any browser toolbar. The ability to search the DBL ,Oklahoma City University (OCU) Law Library catalog, and DBL online databases is an invaluable service. To set up these searches inside the toolbar a URLfor searching the particular catalog or database has to be entered in Conduit ${ }^{\mathrm{TM}}$. The URL can be discovered by performing a catalog or database search and copying the resulting URL. The URL must be edited before use in the toolbar. The original search term(s) will be present in this URL and must be replaced with "MYSEARCHTERM." When you search the DBL catalog and databases information is generated in the URL that is unique to that instance of the search and has to be edited out in order to work properly with Conduit ${ }^{\mathrm{TM}}$. The DBL catalog link that was generated looked like this:

http://library.okcu.edu/cgibin/Pwebrecon.cgi?Search Arg=religion\&SL=None\&S earch_Code=FT*\&PID=F7H9CWoIpt6ziYauOpqWFquxY1xWK\&SEQ=200812 $03092326 \& \mathrm{CNT}=20 \& \mathrm{HIST}=1$. 
Split the URL at the " $\&$ " signs to make it easier to identify specific parts (Figure 1). Normally, the information that needs to be deleted will be anything that is a long string of letters and numbers. In the DBL example the PID and SEQ parts of the URL were removed and the remaining URL was pasted back into the browser, to see if the search worked. Parts continued to be removed from the URL until a working search was conducted with the least amount of parts. Finally, the search term, "religion" was replaced with "MYSEARCHTERM" and the resulting link was pasted into Conduit ${ }^{\mathrm{TM}}$. The final URL was:

http://library.okcu.edu/cgibin/Pwebrecon.cgi?Search_Arg=MYSEARCHTERM\& Search_Code $=$ FT $* \& C N T=25 \& H I S T=1$.

The same steps were performed to add searches for the OCU Law Library catalog search, the OCU web site search, and the DBL EBSCO database search. With any licensed database search. an ezproxy address has to be placed in front of the newly edited search URL to make sure the toolbar is usable off-campus.

\section{Figure 1}

Parts of Search URL

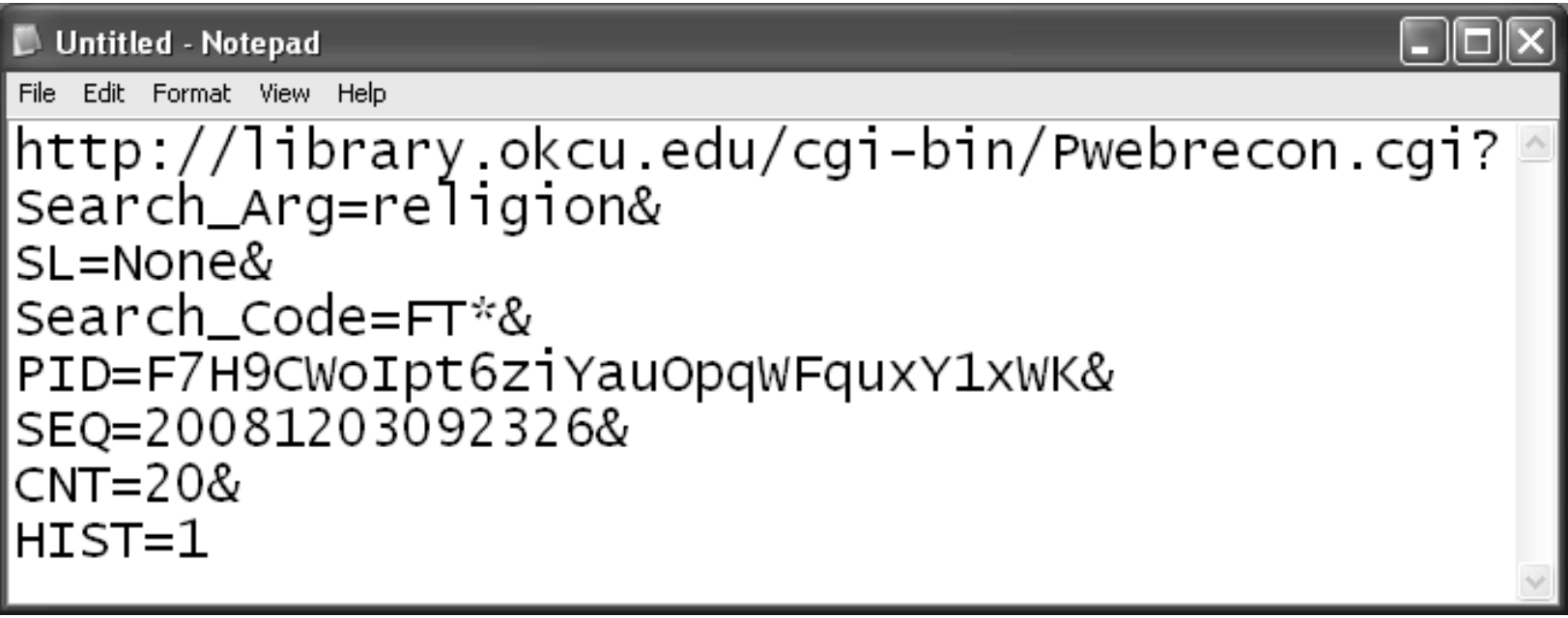

The next step was deciding what other features to include in the toolbar. Links to library research guides, the reserves section of the catalog, and the media search box were added. These three items were chosen because students were often unaware of them. The library toolbar not only makes searching easier for patrons, but it also provides a method for marketing new and underutilized library services. Links are the easiest items to add.. Choose an icon, type a label, input the web address and a new link will be added to the toolbar.

Conduit ${ }^{\mathrm{TM}}$ offers many customizable components including a ticker, menus, RSS feeds, podcasts, and widgets. The Dulaney-Browne Library monthly trivia question was added as a ticker on the toolbar. To create a ticker, choose a size, a text color, and font and type in a message or question. Conduit's ${ }^{\text {TM }}$ interface is point and click. All input items are clearly labeled and pictorial examples are provided. However, new components can be created using the documentation available for developers from the Conduit ${ }^{\mathrm{TM}}$ website (www.conduit.com/Developers/Library/Default.aspx). 
The last thing added to the toolbar was the Dulaney-Browne Library (DBL) logo. This provided a unique opportunity to create brand name recognition for the library. Adding the logo was one of the most challenging development tasks because the DBL logo has text in the shape of a circle. If you shrink the logo small enough to fit on the toolbar, the image is blurry and the text is hard to read. The image part of the logo had to be extracted and placed beside the library's name to make it readable. The other challenge was keeping the text and image from pixelating. The resolution was increased in an attempt to solve this problem, but was unsuccessful. In the end DBL used an image that had 300 pixels per inch resolution and was 660 pixels wide and 75 pixels tall (Figure $2 \& 3$ ). The image is slightly pixilated, but readable.

\section{Figure 2}

Logo used for Library Toolbar

IIII|||Dulaney-Browne Library

\section{Figure 3}

Image of the Library Toolbar

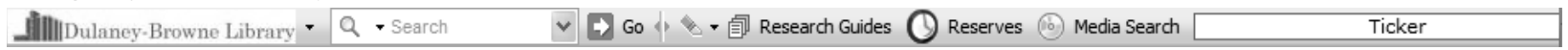

In addition to offering customization options on the toolbar itself, Conduit ${ }^{\mathrm{TM}}$ also allows you to add logos and customized color schemes to the download page (Figure 4) and the search results page (Figure 5), adding additional branding opportunities. One downside to the search results page is that the sponsored sites are listed first. However, they are clearly marked as such so it is very easy to scroll down to non-sponsored sites. Another downside is the inability to make the catalog results appear first. The Google search results always appear first and the user must click on the library catalog, EBSCO, and other customized search links across the top of the page (Figure 4) to view those search results. However, this is a small price to pay for a free toolbar.

\section{Figure 4}

Download Page 

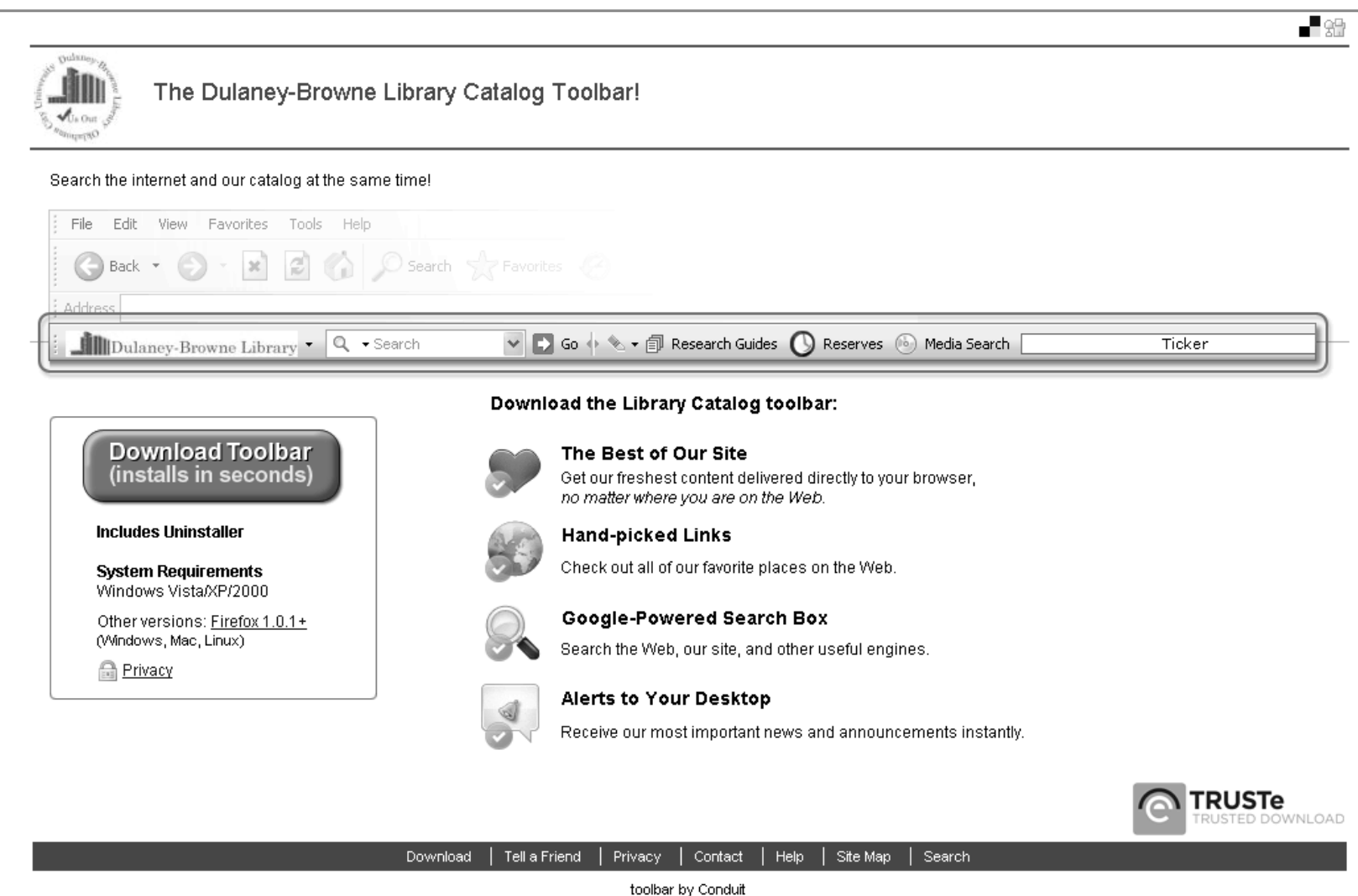

Figure 5

Search Results Page

Dilllibulaney-Browne Library
Web Library Catalog EBSCo ocu Website Law Library Catalog Images More T
Sponsored Results
Chocolate and Candy Gifts
Variety Of Gourmet Candy Gifts Chocolates, Gummies, Fudge \& More
whw. HickoryFarms.com

\section{Compatibility and Implementation}

Once the customization process was finished, the Dulaney-Browne Library (DBL) toolbar was distributed among staff members for testing. The toolbar had only one major problem; it was incompatible with the Ebrary Reader. DBL offers e-books from Ebrary and while the toolbar was enabled, the table of contents for the ebooks would not load in Internet Explorer (IE). A blinking arrow would appear where the table of contents was supposed to be. In addition IE would frequently freeze or shut down. However, the Ebrary Reader works well in Mozilla Firefox with the toolbar enabled. Multiple tests were performed, such as disabling all add-ons except the Ebrary Reader ActiveX control and the library toolbar. The e-books failed to load unless the toolbar was disabled. Ebrary has another reader available that uses Java and 
while it is compatible with the library toolbar, DBL is waiting for IT staff to upgrade the campus MAC computers with the latest version of Java before switching is considered. Both Ebrary and Conduit ${ }^{\mathrm{TM}}$ support have been contacted concerning the compatibility issues. Conduit ${ }^{\mathrm{TM}}$ performed a remote connection to a DBL computer so the problem could be reproduced, but so far a solution has not been found. Because of the compatibility issues with Ebrary, DBL has not yet made the toolbar available to faculty and students. Plans are still underway to distribute the toolbar in the future, once a solution has been found.

Even with the compatibility issues, the overall experience with Conduit ${ }^{\mathrm{TM}}$ has been very positiveamong staff members who use the toolbar constantly. When an Ebrary item is needed either the toolbar is disabled or Mozilla Firefox is used. Before the toolbar is deployed for use by the general public, a chat feature as well as a menu for more links to the Dulaney-Browne Library (DBL) web site will be added. Once it is released, we hope the toolbar will be frequently used by library users and that they associate the helpfulness of the toolbar with the library itself.

You can download the DBL toolbar at http://librarycatalog.ourtoolbar.com. For more information about Conduit ${ }^{\mathrm{TM}}$ services visit their homepage (www.conduit.com) or the community forums (http://forum.conduit-network.com/). Additional applications for the Conduit ${ }^{\mathrm{TM}}$ toolbar are discussed on the Library Toolbar Blog (http://librarytoolbar.blogspot.com/). 


\section{Reference List}

Balas, J.I. 2008. "online treasures." Computers in Libraries 28, no. 5: 52-52. 
Article Type: General Review

Short Biography

Elizabeth Jones (ejones@okcu.edu) is the Access Services and Systems Librarian at the Dulaney-Browne Library, Oklahoma City University, Oklahoma City, Oklahoma, USA.

\begin{abstract}
:
Purpose - The purpose of this article is to review the toolbar creation service, Conduit ${ }^{\mathrm{TM}}$ and provide instruction for configuring the toolbar search box.

Design/Methodology/Approach - This article is based on the experience of the DulaneyBrowne Library while developing a library toolbar using Conduit ${ }^{\mathrm{TM}}$.

Findings - Conduit ${ }^{\mathrm{TM}}$ is a free service for creating toolbars to use with Mozilla Firefox and Internet Explorer. Conduit ${ }^{\mathrm{TM}}$ is user-friendly and intuitive. However, there is a compatibility issue with the Internet Explore version and the Ebrary Reader ActiveX Contorl.

Originality/Value - This paper offers helpful tips on developing a library toolbar.
\end{abstract}

Keywords: Library Toolbars; Conduit;; Web 2.0 